\title{
ИНТЕГРАЦИОННО-МОДУЛЬНЫЙ ПОДХОД К КОНТРОЛЛИНГУ В СИСТЕМЕ УПРАВЛЕНИЯ ПРЕДПРИЯТИЕМ
}

\author{
(c) 2018 Хачемизов Анзор Русланович \\ начальник отдела \\ Акционерное общество «Краснодарпроектстрой» \\ 350909, г. Краснодар, ул. Чапаева, 94 \\ E-mail: zarubin.vi18@yandex.ru
}

В статье рассматривается интеграционно-модульный подход к контроллингу в системе управления предприятием. Приведена интеграционно-модульная структура механизма контроллинга. Обоснована целесообразность внедрения данного подхода на промышленных предприятиях. Определено содержание основных функций и задач контроллинга.

Ключевые слова: контроллинг, система управления предприятием, интеграционно-модульная модель, функционально-процессный модуль.

На современном этапе развития известно достаточно большое количество методологических подходов к формированию контроллинга на предприятии. Широко известны: системный, проектный, процессный, процессно-модульный, функциональный, маркетинговый и др. Множество перечисленных подходов не отражают весь методологический спектр процессов формирования и организации функционирования системы контроллинга. Каждый из подходов является односторонним отражением полиаспектной сущности контроллинга. При этом область исследования (контроллинга на предприятии) объективно сужается вследствие моноаспектности, акцентировании на отдельных свойствах, видах и особенностях контроллинга. Безусловно, система контроллинга обладает определенной специфичностью относительно организационных характеристик и не может не зависеть от отраслевой специфики, способа организации производства, уровня автоматизации и других характеристик предприятия. С другой стороны специфичность системы контроллинга не отвергает возможность унификации процессов и процедур контроллинга [1].

Теория интегрированного контроллинга является методологической основой формирования системы контроллинга, обладающей способностью унификации и регламентации системных процедур и процессов. Использование принципов интеграции позволяет логически согласовать по вертикали и горизонтали действия всех элементов и подсистем контроллинга в увязке со стратегическими приоритетами и требованиями оперативной необходимости.
Основываясь на определении интегрированного контроллинга, целесообразно рассматривать его структуру как совокупность отдельных функционально-процессных модулей (рисунок 1).

Гусева И.Б. идентифицирует интегрированный контроллинг как «процесс объединения и взаимной увязки целей, задач, функций, инструментов, методик отдельных подсистем контроллинга в единый комплекс на основе устойчивых связей информационного обмена» [2]. Известно, что основная цель интеграции элементов контроллинга состоит в повышении эффективности управления предприятием. Основываясь на данном положении в качестве основополагающего принципа формирования системы контроллинга целесообразно выделить закон необходимого разнообразия У.Эшби [3], в соответствии с которым степень разнообразия управляющей системы должна быть не меньше разнообразия управляемой системы. Применительно к контроллингу это выражается в том, что его целевая функция обеспечивает сужение разнообразия контролируемых организационных процессов (среды). В данном случае учитывается, что система контроллинга сформирована и функционирует по типу модели жизнеспособной системы (МЖС) С. Бира [4].

Предполагается, что система контроллинга на предприятии представляет собой иерархически организованный многомодульный комплекс. Каждый модуль сформирован на принципах функционально-процессного подхода, то есть является подсистемой контроллинга или агрегированной функции (планирования, контроля, поддержки решений) или отдельного процесса, 


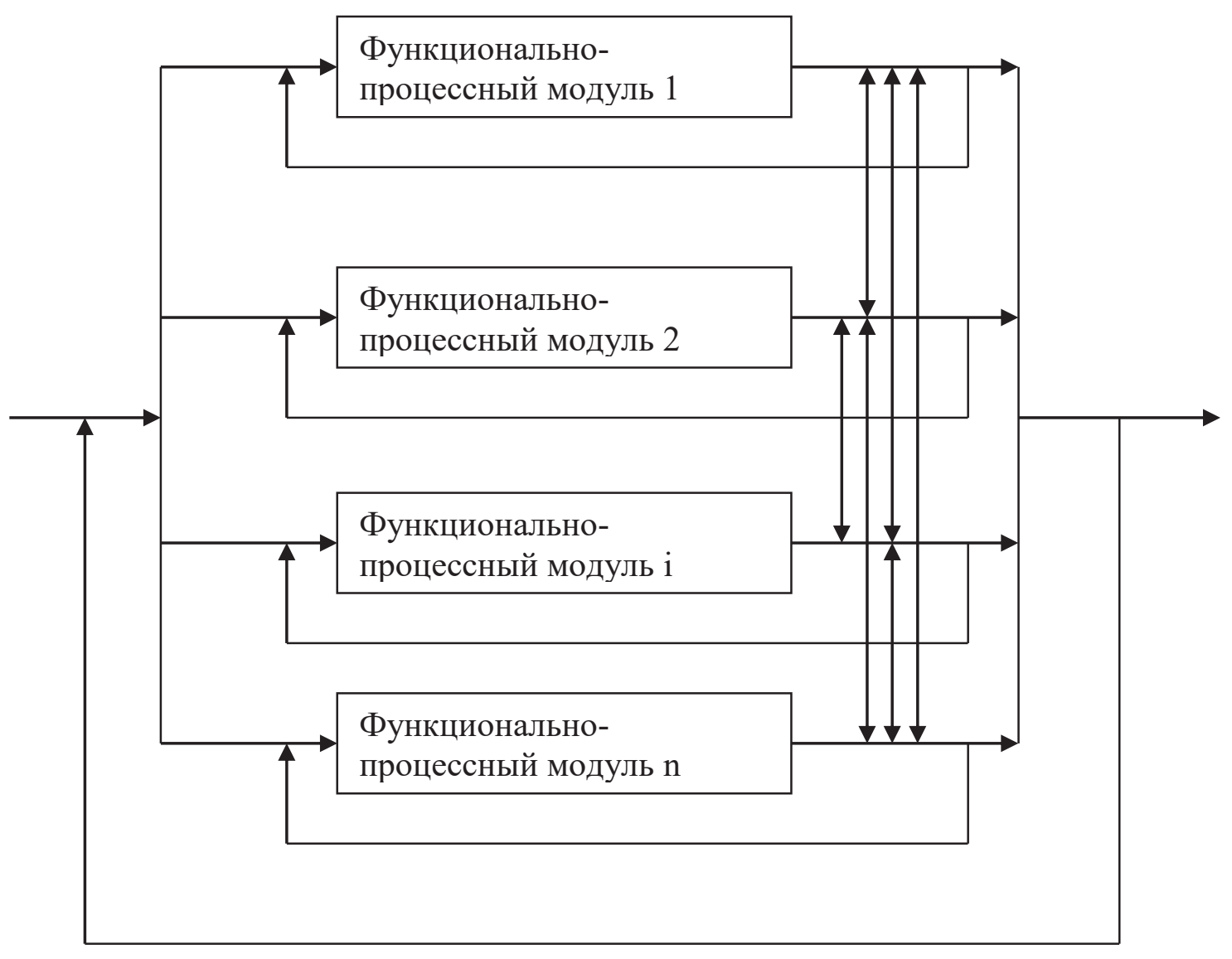

Puc. 1. Интеграционно-модульная модель контроллинга на предприятии

реализуемых на предприятии. Модули могут быть интегрированы как по вертикали, так и по горизонтали организационного управления.

Концептуально организационная система предприятия представлена подсистемами: технологической, финансовой, проектной и социальной. Технологическая подсистема представляет собой технико-технологический комплекс предприятия, включающий совокупность машин, механизмов и технологий, находящихся в соответствующих взаимосвязях и зависимостях, отличающихся разнообразием и характеризуемых показателями с определенными параметрами [5]. В данную подсистему целесообразно включить и подсистему организации рабочих мест. Наблюдение и анализ показателей работы данной подсистемы осуществляются технологическим процессно-функциональным модулем контроллинга.

Финансовая подсистема контроллинга (финансовый процессно-функциональный модуль) ориентирована на мониторинг и контроль параметров и взаимосвязей основного, оборотного и человеческого капитала. Безусловно, важнейшие экономические показатели работы предприятия, такие как доход, себестоимость, прибыль отслеживаются ресурсным контроллингом.

Проектная подсистема контроллинга является механизмом, контролирующим изменения в сфере реализации различных бизнес-процессов. Очевидно, что в данном случае возникает потребность в использовании современных инструментарных средств анализа, методик учета и аналитической обработки параметров и динамики затрат, доходов, рисков и т.д. Успешная реализация многообразия проектов является основополагающим фактором, обеспечивающим эффективность функционирования предприятия.

В свою очередь, социальная подсистема контроллинга подразумевает анализ использования человеческого капитала предприятия [6]. Основное её целевое назначение состоит в анализе параметров и взаимосвязей факторов повышения производительности труда.

Система управления предприятием любой организации представляет собой совокупность интегрированных подсистем управления и 
функционально-процессных модулей контроллинга. В данном контексте система контроллинга обеспечивает обратную связь в процессах управления и регулирования функционирования предприятия.

Методологическую основу системы контроллинга промышленных предприятий составляют цели, задачи, закономерности развития, методы и технология. Данные элементы обеспечивают ее практическую реализацию. Функциональная и организационная структуры, совокупность организационных отношений, системы взаимодействия органов управления и квалификация персонала составляют базис механизма контроллинга. Техническая составляющая механизма контроллинга представлена системой документооборота и организационно-коммуникационной техникой. В этой связи целесообразно полагать, что методологическая основа формирует концепцию контроллинга, а техническая - механизм его реализации. Эффективность системы контроллига обусловливается интеграционной составляющей и базируется на взаимодействии модулей контроллинга.

Интеграционно-модульная структура механизма контроллинга на предприятии представлена на рисунке 2.

Данный подход позволяет в полной мере реализовать основной принцип системы контроллинга на предприятии, заключающийся в «управлении по отклонениям» [7]. При этом реализуются четыре основных функции: планирование; контроль и анализ; информационная; инструментарная. Все названные функции реализуются в различных модулях одновременно. Так, планирование осуществляется в процессе функционирования четырех основных процессно-функциональных модулей контроллинга:

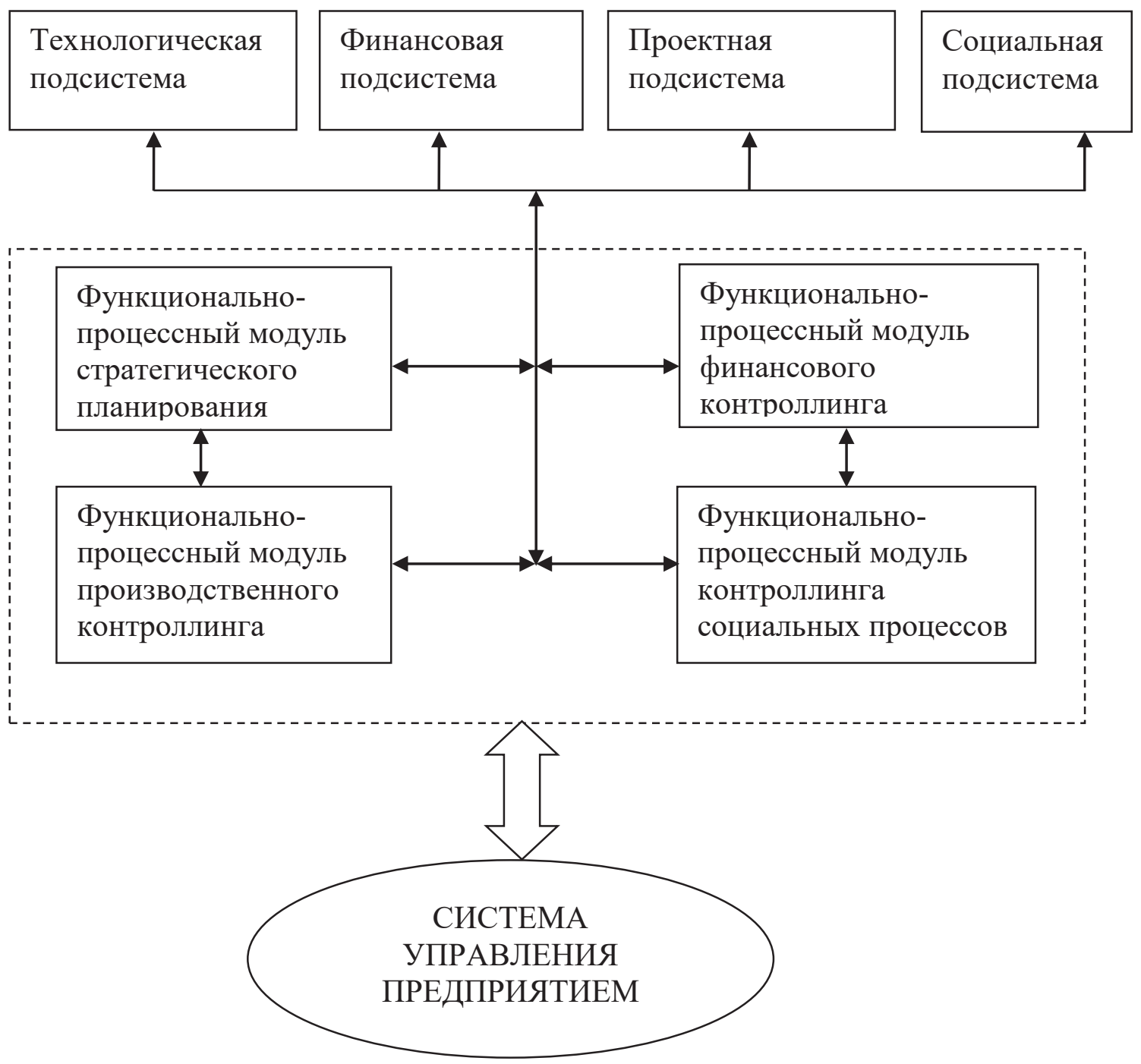

Puc. 2. Интегрально-модульная структура механизма контроллинга на предприятии 
технологическом, финансовом, проектном и социальном. Например, задача разработки стратегии предприятия и контроль за её реализацией может быть решена при условии комплексного анализа процессов функционирования различных подсистем предприятия. Не менее важной является задача тактического и оперативного планирования, решение которых связано с анализом показателей работы предприятия в различны аспектах его деятельности.

Инструментарная функция механизма контроллинга подразумевает его реализацию в роли поддержки принятия решений, позволяющей в полной мере реализовать управленческие функции и оптимизировать систему управления предприятием [8]. В этой связи, система контроллинга собой комплексный методико-информационно-аналитический инструмент, направленный на реализацию управленческих функций. Целесообразно рассматривать данную модульную составляющую в виде подсистемы управления предприятием, выделяющейся в отдельное структурное подразделение, занимающееся также вопросами повышения конкурентоспособности предприятия и улучшения качества продукции. К задачам данного элемента системы управления относятся:

- комплексное методическое обеспечение всех подсистем контроллинга;

- выявление и анализ технологических, производственных и эксплуатационных проблем и отклонений показателей от целевых значений;

- нормоконтроль продукции, сырья, материалов;

- контроль качества инструментария и оснащения предприятия.

Эффективность данной контроллинговой составляющей зависит от качества реализации ее функционала $[9,10]$.

Еще одной неотъемлемой функцией механизма контроллинга на предприятии является информационная, реализующая мониторинг технологических, воспроизводственных и социальных процессов процессов. Данная система периодического сбора, обобщения и оценки информации в совокупности с остальными модулями контроллинга позволяет наиболее эффективно и комплексно подходить к вопросам принятия и реализации управленческих решений на всех уровнях, с учетом специфики деятельности предприятия для повышения его эффективности. Данный элемент позволяет обеспечить высокий уровень управления процессами, как на предприятии, так и за его пределами, но непосредственно оказывающими влияние на его функционирование. В основу реализации информационной функции контроллинга положен принцип получения системной и достоверной информации, скорость ее распределения, актуальность полученных данных и единая методология сбора [11].

Для реализации функции контроля и анализа финансовой деятельности механизмом контроллинга используется система показателей, позволяющая осуществить оценку рациональности использования финансовых ресурсов. Основная цель финансового контроля заключается в расчете прибыли и рентабельности возврата инвестиций за определенный временной период в соотношении к планированию деятельности предприятия и его стратегической ориентации [12].

Основным источником получения информации для реализации функции контроля и анализа является бухгалтерская отчетность предприятия. На его основе проводится оценка эффективности функционирования предприятия. Контроль финансовой деятельности позволяет своевременно выявлять недочеты и проблемы в финансовом секторе предприятия для их оперативного устранения.

Интеграция функционально-процессных модулей контроллинга позволяет в реальном времени отслеживать и контролировать не только основные процессы, протекающие в рамках предприятия, но и анализировать и прогнозировать возможные последствия от действия различных внешних факторов. Данная система управленческой поддержки способствует качественно новому уровню принятия решений с учетом удовлетворения всех потребностей и достижению текущих и стратегических целей предприятия.

Интеграционно-модульный подход позволяет решать следующие управленческие задачи, возникающие вследствие особых свойств социально-экономических систем:

- структурирование процесса управления с целью выделения базовых, сервисных и промежуточных модулей как носителей системных свойств, совершенствование управления которыми в контексте заданного направления развития позволяет повысить эффективность при- 
нятия решений;

- анализ и алгоритмизация управленческих функций для системы в целом и отдельных модулей;

- разработка целевых функций, программ и проектов по их реализации на основе методов анализа иерархий;

- трансформация структуры принятия и реализации решений, в том числе формирования адаптивных структур управления;

- разработка инструментария анализа функционирования усовершенствованной структуры системы в контексте управления.

Формирование системы контроллинга с учетом интеграционно-модульного подхода на предприятии позволит адекватно и своевременно реагировать на все изменения и погрешности, обеспечивая достижение поставленных целей. Необходимо отметить и универсальность применения данного подхода, что позволяет обеспечить высокую его эффективность на предприятиях любой формы собственности.

Механизм контроллинга является по существу инструментарным базисом системы управления, способствующий наиболее эффективному стратегическому развитию предприятия на основе анализа ситуации. Основной принцип контроллинга состоит в осуществлении эффективного управления предприятием с учетом отклонений. В сфере обеспечения стратегической стабильности основная задача контроллинга предприятия состоит в разработке эффективных управленческих воздействий для минимизации отклонений развития, вызванных внуренними и внешними проявлениями.

Применение системы контроллинга на предприятиях способствует оперативному устранению проблем, возникающих при воздействии внутренних и внешних раздражителей, способствует эффективному решению задач информационно-координационного блока и осуществляет всестороннюю поддержку систем управления с учетом специфики ее функционала [13]. Практическая реализация системы контроллинга предприятия состоит в диагностике кризисного состояния деятельности предприятия, выявлении основных направлений его развития для предотвращения негативных проявлений. Для оценки эффективности внедрения механизма контроллинга, разработанного на основе инегрционно-модульного подхода необходимо установить соотношение затрат на внедрение контроллинга с полученными результатами от мероприятий, основанных на его практическом применении.

Интеграционно-модульный подход в контроллинге позволяет использовать интеграционную составляющую в процессах принятия решений. Даная система обладает высокой адаптивностью и может успешно применяться на предприятиях всех форм собственности. Система позволяет стабилизировать процессы, протекающие внутри предприятия и оказать существенное влияние на внешние раздражители.

Таким образом, создание системы контроллинга на основе интеграционно-модульного подхода обеспечивает принятие оптимальных решений в процессах управления предприятием. Это обусловливается применением современных методов и инструментов, позволяющих прогнозировать потенциальные угрозы и разрабатывать тактически верные управленческие решения с учетом стратегии развития предприятия, повысить мобильность управляющих воздействий.

\section{Библиографический список}

1. Ивашкевич В.Б. Контроллинг: сущность и назначение// Бухгалтерский учет. 1991. № 7. С. 812.

2. Гусева И.Б., Калугина Т.В. Интегрированное управление ресурсами, процессами и результатами в системе контроллинга // Контроллинг. 2011. № 1(38).

3. Эшби У.Р. Введение в кибернетику. Москва. 1959.

4. Beer S. Diagnosing the System for Organisations. Wiley, Chichester, 1990.

5. Фатхутдинов Р. А. Производственный менеджмент: учебник для вузов. Санкт-Петербург. 4-е изд. 2003.

6. Дайле А. Практика контроллинга. Москва. 2001.

7. Лестер Р. Биттель. Управление по отклонениям. Москва. 1999.

8. Карминский А.М., Оленев Н.И., Примак А.Г., Фалько С.Г. Контроллинг в бизнесе. Методологические и практические основы построения контроллинга в организациях. Москва. 2-е изд. 2002.

9. Стуков С.А. Система производственного учета и контроля. Москва.1988.

10. Фольмут Х.И. Инструменты контроллинга. Москва. 1998. 
11. Исаева О.В. Модель структуры и процесса функционирования организационно-экономического механизма контроллинга предприятия // Экономинфо, 2005. № 4 .

12. Бурцев В.В. Контроллинг финансов: варианты и направления // Менеджмент сегодня. 2003. № 5.

13. Швейкерт М.И. Проектно-блочная схема формирования контроллинга в промышленной корпорации // Интеллект. Инновации. Инвестиции. 2011. № 2.

Поступила в редакциюю 28.08.2018 